\title{
Low-Frequency Vibration Modes of Strongly Inhomogeneous Elastic Laminates
}

\begin{abstract}
J. D. Kaplunov, L. A. Prikazchikova
Julius D. Kaplunov, https://orcid.org/0000-0001-7505-4546, School of Computing and Mathematics, Keele University, Keele, Staffordshire, ST5 5BG, UK, j.kaplunov@ keele.ac.uk

Ludmila A. Prikazchikova, https://orcid.org/0000-0001-9051-2103, School of Computing and Mathematics, Keele University, Keele, Staffordshire, ST5 5BG, UK, I.prikazchikova@ keele.ac.uk

The dynamic behaviour of thin multi-layered structures, composed of contrasting "strong" and "weak" layers, is considered. An asymptotic procedure for analysing the lowest cutoffs is developed. A polynomial frequency equation is derived, along with the linear equations for the associated eigenforms corresponding to displacement variation across the thickness. For a five-layered laminate with clamped faces two term expansions for eigenfrequencies and eigenforms are compared with those obtained from the exact solution of the original problem for thickness resonances.
\end{abstract}

Key words: asymptotic, high-contrast, thin elastic structure, thickness fluctuations, low frequency, cutoff.

DOI: https://doi.org/10.18500/1816-9791-2018-18-4-447-457

\section{INTRODUCTION}

The classical theory for thin homogeneous elastic structures governing bending, extensional and torsional-shear vibration modes, e.g. see [1-3], is asymptotically consistent over the low-frequency range. Obviously, it does not take into consideration any of high-frequency modes with the cut-off frequencies related to thickness resonances. At the same time, currently available refined formulations incorporating the lowest high-frequency modes ( $[4,5]$ and reference therein) do not appear to be uniformly asymptotically valid, as noted for example in [6]. These observations are also true for layered structures not demonstrating a substantial contrast in material and geometric properties of the layers, e.g. [7]. However, multi-parametric analysis of strongly vertical inhomogeneous three-layered plates in [6], see also [8] and [9], indicates that for certain combination of problem parameters the lowest thickness shear resonance frequency becomes asymptotically small, resulting in an extra low-frequency vibration mode in comparison with the traditional layout. Four scenarios studied in [6] correspond to the practically important setups of sandwich structures, laminated glass, photovoltaic panels and precipitator plates utilised in gas filters, see [10-13].

In this paper we extend the framework of the cited publication [6] on thin strongly inhomogeneous plates to multi-layered structures with arbitrary number of layers. For the sake of definiteness, the upper and lower faces are supposed to be clamped. For homogeneous plates, such boundary conditions do not support low-frequency vibrations $[14,15]$. Below we consider layers of two different types, namely, "strong" and "weak" ones, adapting the ratio of their stiffnesses and densities as a single small parameter, such that the wave speeds in strong and weak components are of the same order. In addition, we do not impose any special restriction on the thicknesses of the layers, apart from the assumption that all of them are also of the same asymptotic order. The consideration is restricted to the evaluation of thickness resonances.

The paper is organised as follows. The problem is formulated in Section 1. The general asymptotic procedure is developed in Section 2. A polynomial equation is derived for the 
leading order values of the thickness resonances along with linear algebraic equations for the associated eigenforms. As might be expected, the order of the aforementioned equation coincides with the number of strong layers undergoing almost rigid body motion [9]. In this case the weaker layers are subject to nearly homogeneous thickness deformation. The results of Section 2 are specified in the next Section 3 for a five-layered laminate. A correction to the leading order approximation is also calculated, in order to illustrate small deviations from rigid body motions and homogeneous deformations. Numerical results are discussed in the last section.

\section{STATEMENT OF THE PROBLEM}

Consider a thin elastic laminate composed of $n$ alternating strong and weak layers of thickness $h_{i}, i=1, \ldots, n$, see Fig. 1 . Denote Young moduli, Poisson ratios and densities of the layers by $E_{m}, \nu_{m}$ and $\rho_{m}$ with $m=1$ and $m=2$ corresponding to weak and strong layers, respectively. Thus, $E_{1} \ll E_{2}$ and also, for the sake of definiteness, we assume that $E_{1} / E_{2} \sim \rho_{1} / \rho_{2}$.

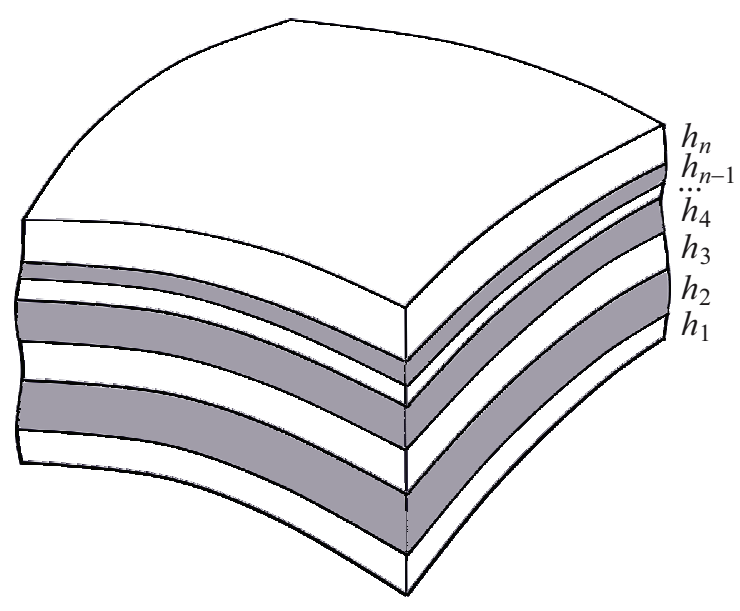

Fig. 1. Multi-layered laminate with $n$ alternating weak and strong layers

The consideration below is oriented to harmonic vibrations with angular frequency $\omega$ of a laminate with fixed faces over the low frequency range

$$
\omega \ll \frac{1}{h_{i}} \sqrt{\frac{E_{m}}{\rho_{m}}}, \quad i=1, \ldots, n, \quad m=1,2 .
$$

The main focus is on the effect of elastic contrast, since low frequency vibrations is not a feature of homogeneous structures with fixed faces, see [14]. We restrict ourselves to the evaluation of the cutoffs of lowfrequency modes arising near the thickness resonances of a flat plate clamped along the faces of weak outer layers.

We start from the equations [2]

$$
\frac{E_{m}}{2\left(1+\nu_{m}\right) \varkappa_{m}} \frac{\mathrm{d}^{2} v_{3 i}}{\mathrm{~d} z^{2}}+\rho_{m} \omega^{2} v_{3 i}=0, \quad i=1, . ., n, \quad m=1,2,
$$

where

$$
\varkappa_{m}=\sqrt{\frac{1-2 \nu_{m}}{2-2 \nu_{m}}}
$$

and

$$
\frac{E_{m}}{2\left(1+\nu_{m}\right)} \frac{\mathrm{d}^{2} v_{k i}}{\mathrm{~d} z^{2}}+\rho_{m} \omega^{2} v_{k i}=0, \quad k=1,2,
$$

for stretch and shear thickness vibrations, respectively, with $v_{k i}$ and $v_{3 i}$ denoting tangential and transverse displacements. The continuity conditions along interfaces are written as

$$
v_{3 i}=v_{3 j}, \quad \frac{E_{1}}{2\left(1+\nu_{1}\right) \varkappa_{1}} \frac{\mathrm{d} v_{3 i}}{\mathrm{~d} z}=\frac{E_{2}}{2\left(1+\nu_{2}\right) \varkappa_{2}} \frac{\mathrm{d} v_{3 j}}{\mathrm{~d} z}
$$


or

$$
\begin{aligned}
& v_{k i}=v_{k j}, \quad k=1,2, \\
& \frac{E_{1}}{2\left(1+\nu_{1}\right)} \frac{\mathrm{d} v_{k i}}{\mathrm{~d} z}=\frac{E_{2}}{2\left(1+\nu_{2}\right)} \frac{\mathrm{d} v_{k j}}{\mathrm{~d} z},
\end{aligned}
$$

where $j=i+1$ for a "weak-strong" interface and $j=i-1$ for a "strong-weak" one. In addition, along fixed faces $z=0$ and $z=h_{1}+h_{2}+\ldots+h_{n}$

$$
v_{k 1}=v_{31}=v_{k n}=v_{3 n}=0, \quad k=1,2 .
$$

The equations above can be re-written in the form

$$
D_{m} \frac{\mathrm{d}^{2} u_{i}}{\mathrm{~d} z^{2}}+\rho_{m} \omega^{2} u_{i}=0
$$

with

$$
\begin{aligned}
& u_{i}=u_{j}, \\
& D_{1} \frac{\mathrm{d} u_{i}}{\mathrm{~d} z}=D_{2} \frac{\mathrm{d} u_{j}}{\mathrm{~d} z}
\end{aligned}
$$

and

$$
u_{1}=u_{n}=0
$$

at $z=0$ and $z=h_{1}+h_{2}+\ldots+h_{n}$, where $u_{i}=v_{3 i}$ and $D_{m}=\frac{E_{m}}{2\left(1+\nu_{m}\right) \varkappa_{m}}$ for stretch vibrations and $u_{i}=v_{k i}$ and $D_{m}=\frac{E_{i}}{2\left(1+\nu_{i}\right)}$ for shear vibrations.

The formulated boundary value problem can be treated asymptotically over lowfrequency range (1), due to the contrast properties of the layers.

\section{ASYMPTOTIC PROCEDURE}

First, re-write the equations in the previous section in dimensionless local coordinates $Z_{i}=z / h_{i}$ and frequencies $\Omega_{i}=\omega h_{i} / c_{m}$ with $c_{m}=\sqrt{D_{m} / \rho_{m}}, i=1,2, \ldots, n$ and $m=1,2$, having

$$
\frac{\mathrm{d}^{2} u_{i}}{\mathrm{~d} Z_{i}^{2}}+\Omega_{i}^{2} u_{i}=0
$$

The frequency parameters corresponding to both weak or strong $i$-th and $j$-th layers are related to each other as

$$
\Omega_{i}=L_{j}^{i} \Omega_{j}
$$

where $L_{j}^{i}=h_{i} / h_{j}$. At the same time, for $i$-th weak layer and $j$-th strong one

$$
\Omega_{i}=c L_{j}^{i} \Omega_{j},
$$

where $c=c_{2} / c_{1}$. Boundary conditions (4) become

$$
\left.u_{1}\right|_{Z_{1}=0}=\left.u_{n}\right|_{Z_{n}=b_{n}+1}=0,
$$

where

$$
b_{1}=0 \quad \text { and } \quad b_{i}=\frac{1}{h_{i}} \sum_{n=0}^{i-1} h_{n}, \quad i=2, \ldots, n,
$$

such that $b_{i} \leqslant Z_{i} \leqslant b_{i}+1$. 
Continuity conditions (3) along interfaces take the form

$$
\left.u_{i}\right|_{Z_{i}=b_{i}+1}=\left.u_{i+1}\right|_{Z_{i+1}=b_{i+1}}
$$

and

$$
\left.\frac{\mathrm{d} u_{i}}{\mathrm{~d} Z_{i}}\right|_{Z_{i}=b_{i}+1}=\left.\varepsilon^{p} L_{i+1}^{i} \frac{\mathrm{d} u_{i+1}}{\mathrm{~d} Z_{i+1}}\right|_{Z_{i+1}=b_{i+1}},
$$

where $p=1$ if $i$-th layer is strong, and $p=-1$ if it is weak. Here small parameter $\varepsilon$ is introduced as the ratio

$$
\varepsilon=\frac{D_{1}}{D_{2}} \ll 1
$$

As it has been already mentioned, we also assume that $\rho_{1} / \rho_{2} \sim \varepsilon$. In this case we have $\Omega_{1}^{2} \sim \Omega_{2}^{2} \sim \ldots \sim \Omega_{n}^{2} \sim \varepsilon$ over low frequency range (1). Similarly to [9], we expand frequencies $\Omega_{i}$ and displacements $u_{i}$ in the asymptotic series

$$
\Omega_{i}^{2}=\varepsilon\left(\Omega_{i 0}^{2}+\varepsilon \Omega_{i 1}^{2}+\ldots\right)
$$

and

$$
u_{i}=u_{i 0}+\varepsilon u_{i 1}+\ldots
$$

At leading order, we have from equation (5) for strong layers

$$
\frac{\mathrm{d}^{2} u_{i 0}}{\mathrm{~d} Z_{i}^{2}}=0
$$

subject to the Neumann boundary conditions

$$
\left.\frac{\mathrm{d} u_{i 0}}{\mathrm{~d} Z_{i}}\right|_{Z_{i}=b_{i}}=\left.\frac{\mathrm{d} u_{i 0}}{\mathrm{~d} Z_{i}}\right|_{Z_{i}=b_{i}+1}=0 .
$$

As a result, we arrive at uniform variation across the thickness

$$
u_{i 0}=C_{i, 0}=\text { const, }
$$

corresponding to rigid body motion.

Next, we proceed with equations (11) for weak layers. For inner weak layers we have

$$
\left.u_{i 0}\right|_{Z_{i}=b_{i}}=C_{i-1,0},\left.\quad u_{i 0}\right|_{Z_{i}=b_{i}+1}=C_{i+1,0},
$$

while for outer ones

$$
\left.u_{10}\right|_{Z_{1}=0}=\left.u_{n 0}\right|_{Z_{n}=b_{n}+1}=0 .
$$

Thus, we obtain for eigenforms

$$
\begin{aligned}
& u_{10}=C_{2,0} Z_{1}, \\
& \vdots \\
& u_{i 0}=C_{i-1,0}+\left(C_{i+1,0}-C_{i-1,0}\right)\left(Z_{i}-b_{i}\right), \\
& \vdots \\
& u_{n 0}=C_{n-1,0}\left(b_{n}+1-Z_{n}\right) .
\end{aligned}
$$


At next order, we start again with the equations for strong layers following from (5). They are

$$
\frac{\mathrm{d}^{2} u_{i 1}}{\mathrm{~d} Z_{i}^{2}}+\Omega_{i 0}^{2} u_{i 0}=0
$$

The associated boundary conditions, derived from (10) and (12), become

$$
\begin{aligned}
& \left.\frac{\mathrm{d} u_{i 1}}{\mathrm{~d} Z_{i}}\right|_{Z_{i}=b_{i}}=L_{i-1}^{i}\left(C_{i, 0}-C_{i-2,0}\right), \\
& \left.\frac{\mathrm{d} u_{i 1}}{\mathrm{~d} Z_{i}}\right|_{Z_{i}=b_{i}+1}=L_{i+1}^{i}\left(C_{i+2,0}-C_{i, 0}\right) .
\end{aligned}
$$

The compatibility of equations (14) and boundary conditions (15) results in the relations

$$
\begin{aligned}
& C_{2,0} \Omega_{20}^{2}=L_{1}^{2} C_{2,0}-L_{3}^{2}\left(C_{4,0}-C_{2,0}\right), \\
& \vdots \\
& C_{i, 0} \Omega_{i 0}^{2}=L_{i-1}^{i}\left(C_{i, 0}-C_{i-2,0}\right)-L_{i+1}^{i}\left(C_{i+2,0}-C_{i, 0}\right), \\
& \vdots \\
& C_{n-1,0} \Omega_{n-10}^{2}=L_{n}^{n-1} C_{n-1,0}+L_{n-2}^{n-1}\left(C_{n-1,0}-C_{n-3,0}\right) .
\end{aligned}
$$

The latter together with formulae $\Omega_{i 0}=L_{j}^{i} \Omega_{j 0}$, see (6), can be used to determine unknown constants $C_{i, 0}$ and, therefore, leading order eigenfrequencies as will be demonstrated below for a five-layered laminate. In addition, next order corrections to eigenfrequencies and eigenforms will be derived.

\section{FIVE-LAYERED LAMINATE}

Consider a five-layered laminate with alternating weak and strong layers clamped along the faces of outer weak layers, see Fig. 2.

First, we have from (16) for two strong components

$$
\begin{aligned}
& C_{2,0} \Omega_{20}^{2}=L_{1}^{2} C_{2,0}-L_{3}^{2}\left(C_{4,0}-C_{2,0}\right), \\
& C_{4,0} \Omega_{40}^{2}=L_{5}^{4} C_{4,0}+L_{3}^{4}\left(C_{4,0}-C_{2,0}\right),
\end{aligned}
$$

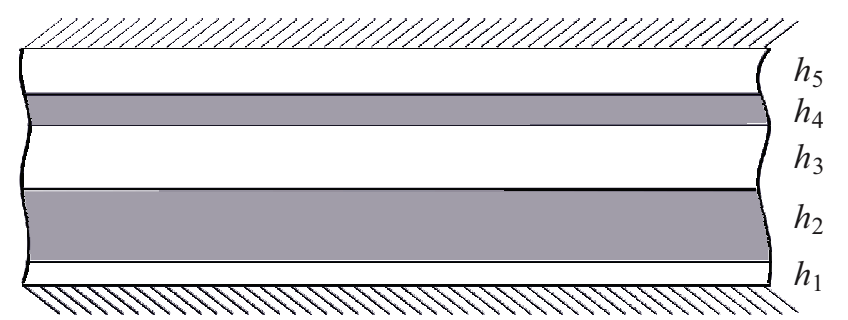

Fig. 2. Five-layered laminate with clamped faces composed of two strong and three weak layers

with $\Omega_{40}=L_{2}^{4} \Omega_{20}$. These equations yield

$$
\Omega_{20}^{2}=\left(L_{4}^{2}\right)^{2}\left(L_{5}^{4}+L_{3}^{4}(1-k)\right),
$$

together with the relation

$$
C_{2,0}=k C_{4,0},
$$

with parameter $k$ defined as

$$
k_{1,2}=\frac{-b \pm \sqrt{b^{2}+4 L_{2}^{4}\left(L_{3}^{4}\right)^{2}}}{2 L_{3}^{4}}, \quad b=L_{1}^{4} L_{2}^{4}+L_{2}^{4} L_{3}^{4}-L_{3}^{4}-L_{5}^{4} .
$$


Then, we obtain from (13) and (18) for leading order eigenforms

$$
\begin{gathered}
u_{10}=C_{4,0} k Z_{1}, \quad u_{20}=C_{4,0} k, \quad u_{30}=C_{4,0}\left(k+(1-k)\left(Z_{3}-b_{3}\right)\right), \\
u_{40}=C_{4,0}, \quad u_{50}=C_{4,0}\left(1+b_{5}-Z_{5}\right) .
\end{gathered}
$$

Next, we have from boundary value problem (14)-(15) for the first order correction to strong layer displacements

$$
u_{i 1}=A_{i, 1} Z_{i}^{2}+B_{i, 1} Z_{i}+C_{i, 1}, \quad i=2,4,
$$

where

$$
\begin{aligned}
A_{2,1} & =-\frac{1}{2} C_{4,0} k \Omega_{20}^{2}, & B_{2,1} & =C_{4,0} k\left(L_{1}^{2}+\Omega_{20}^{2} b_{2}\right), \\
A_{4,1} & =-\frac{1}{2} C_{4,0} \Omega_{20}^{2}\left(L_{2}^{4}\right)^{2}, & B_{4,1} & =C_{4,0}\left(\Omega_{20}^{2}\left(L_{2}^{4}\right)^{2}\left(b_{4}+1\right)-L_{5}^{4}\right) .
\end{aligned}
$$

For weak layers the first order correction is determined from equations (14) subject to the boundary conditions

$$
\begin{array}{ll}
\left.u_{11}\right|_{Z_{1}=0}=0, & \left.u_{11}\right|_{Z_{1}=1}=\left.u_{21}\right|_{Z_{2}=b_{2}}, \\
\left.u_{31}\right|_{Z_{3}=b_{3}}=\left.u_{21}\right|_{Z_{2}=b_{2}+1}, & \left.u_{31}\right|_{Z_{3}=b_{3}+1}=\left.u_{41}\right|_{Z_{4}=b_{4}}, \\
\left.u_{51}\right|_{Z_{5}=b_{5}}=\left.u_{41}\right|_{Z_{4}=b_{4}+1}, & \left.u_{51}\right|_{Z_{5}=b_{5}+1}=0 .
\end{array}
$$

As a result, we arrive at

$$
u_{i 1}=F_{i, 1} Z_{i}^{3}+G_{i, 1} Z_{i}^{2}+H_{i, 1} Z_{i}+K_{i, 1}, \quad i=1,3,5,
$$

where

$$
\begin{aligned}
F_{1,1} & =-\frac{1}{6} C_{4,0} \Omega_{20}^{2}\left(L_{2}^{1}\right)^{2} c^{2} k, & G_{1,1} & =0, \\
F_{3,1} & =\frac{1}{6} C_{4,0} \Omega_{20}^{2}\left(L_{2}^{3}\right)^{2} c^{2}(k-1), & G_{3,1} & =\frac{1}{2} C_{4,0} \Omega_{20}^{2}\left(L_{2}^{3}\right)^{2} c^{2}\left(b_{3}(1-k)-k\right), \\
F_{5,1} & =\frac{1}{6} C_{4,0} \Omega_{20}^{2}\left(L_{2}^{5}\right)^{2} c^{2}, & G_{5,1} & =-\frac{1}{2} C_{4,0} \Omega_{20}^{2}\left(L_{2}^{5}\right)^{2} c^{2}\left(b_{5}+1\right) .
\end{aligned}
$$

Then, setting one of the constants equal to zero, namely $C_{4,1}=0$, and applying Dirichlet boundary conditions for weak layers we obtain for the rest of the constants

$$
\begin{gathered}
H_{1,1}=A_{2,1} b_{2}^{2}+B_{2,1} b_{2}-F_{1,1}+C_{2,1} \\
H_{3,1}=A_{4,1} b_{4}^{2}+B_{4,1} b_{4}-A_{2,1}\left(b_{2}+1\right)^{2}-B_{2,1}\left(b_{2}+1\right)-F_{3,1}\left(3 b_{3}^{2}+3 b_{3}+1\right)- \\
-G_{3,1}\left(2 b_{3}+1\right)-C_{2,1} \\
H_{5,1}=-A_{4,1}\left(b_{4}+1\right)^{2}-B_{4,1}\left(b_{4}+1\right)-F_{5,1}\left(3 b_{5}^{2}+3 b_{5}+1\right)-G_{5,1}\left(2 b_{5}+1\right)
\end{gathered}
$$

and

$$
\begin{gathered}
K_{1,1}=0, \quad K_{3,1}=A_{4,1} b_{4}^{2}+B_{4,1} b_{4}-F_{3,1}\left(b_{3}+1\right)^{3}-G_{3,1}\left(b_{3}+1\right)^{2}-H_{3,1}\left(b_{3}+1\right), \\
K_{5,1}=-F_{5,1}\left(b_{5}+1\right)^{3}-G_{5,1}\left(b_{5}+1\right)^{2}-H_{5,1}\left(b_{5}+1\right),
\end{gathered}
$$

where $C_{2,1}$ is still unknown. 
Now, we consider vibrations of strong layers at second order, starting from the equations

$$
\frac{\mathrm{d}^{2} u_{i 2}}{\mathrm{~d} Z_{i}^{2}}+\Omega_{i 0}^{2} u_{i 1}+\Omega_{i 1}^{2} u_{i 0}=0, \quad i=2,4,
$$

subject to the boundary conditions

$$
\begin{aligned}
& \left.\frac{d u_{i 2}}{d Z_{i}}\right|_{Z_{i}=b_{i}}=\left.L_{i-1}^{i} \frac{d u_{i-11}}{d Z_{i-1}}\right|_{Z_{i-1}=b_{i-1}+1}, \\
& \left.\frac{d u_{i 2}}{d Z_{i}}\right|_{Z_{i}=b_{i}+1}=\left.L_{i+1}^{i} \frac{d u_{i+11}}{d Z_{i+1}}\right|_{Z_{i+1}=b_{i+1}} .
\end{aligned}
$$

The compatibility condition for the last boundary value problem leads to

$$
\begin{aligned}
\Omega_{21}^{2} & =\frac{1}{6 k}\left(2 A_{2,1}^{\star}\left(-3 b_{2}^{2} \Omega_{20}^{2}+3 L_{1}^{2} b_{2}^{2}+3 L_{3}^{2} b_{2}^{2}-3 b_{2} \Omega_{20}^{2}+3 L_{3}^{2}+6 L_{3}^{2} b_{2}-\Omega_{20}^{2}\right)-\right. \\
-6 & A_{4,1}^{\star} L_{3}^{2} b_{4}^{2}+3 B_{2,1}^{\star}\left(-2 b_{2} \Omega_{20}^{2}+2 L_{1}^{2} b_{2}+2 L_{3}^{2} b_{2}-\Omega_{20}^{2}+2 L_{3}^{2}\right)-6 B_{4,1}^{\star} L_{3}^{2} b_{4}+ \\
& \left.+6 C_{2,1}^{\star}\left(-\Omega_{20}^{2}+L_{1}^{2}+L_{3}^{2}\right)+12 F_{1,1}^{\star} L_{1}^{2}+6 F_{3,1}^{\star} L_{3}^{2}\left(3 b_{3}+\right)+6 G_{3,1}^{\star} L_{3}^{2}\right)
\end{aligned}
$$

where

$$
\begin{gathered}
C_{2,1}^{\star}=\frac{C_{2,1}}{C_{4,0}}=-\frac{1}{6} \frac{1}{L_{1}^{4} L_{2}^{4}+L_{2}^{4} L_{3}^{4}+L_{3}^{4} k-\left(L_{2}^{4}\right)^{2} \Omega_{20}^{2}} \times \\
\times\left(2 A _ { 2 , 1 } ^ { \star } \left(3 L_{1}^{4} L_{2}^{4} b_{2}^{2}+3 L_{2}^{4} L_{3}^{4} b_{2}^{2}-3 b_{2}^{2} \Omega_{20}^{2}\left(L_{2}^{4}\right)^{2}-3 b_{2} \Omega_{20}^{2}\left(L_{2}^{4}\right)^{2}+6 L_{2}^{4} L_{3}^{4} b_{2}-\right.\right. \\
\left.-\Omega_{20}^{2}\left(L_{2}^{4}\right)^{2}+3 L_{3}^{4} b_{2}^{2} k+3 L_{2}^{4} L_{3}^{4}+6 L_{3}^{4} b_{2} k+3 L_{3}^{4} k\right)+2 A_{4,1}^{\star}\left(3 b_{4}^{2} k \Omega_{20}^{2}\left(L_{2}^{4}\right)^{2}+\right. \\
\left.+3 b_{4} k \Omega_{20}^{2}\left(L_{2}^{4}\right)^{2}-3 L_{2}^{4} L_{3}^{4} b_{4}^{2}+k \Omega_{20}^{2}\left(L_{2}^{4}\right)^{2}-3 L_{3}^{4} b_{4}^{2} k-3 L_{5}^{4} b_{4}^{2} k-6 L_{5}^{4} b_{4} k-3 L_{5}^{4} k\right)- \\
-3 B_{2,1}^{\star}\left(2 b_{2} \Omega_{20}^{2}\left(L_{2}^{4}\right)^{2}+2 L_{1}^{4} L_{2}^{4} b_{2}+6 L_{2}^{4} L_{3}^{4} b_{2}-\Omega_{20}^{2}\left(L_{2}^{4}\right)^{2}+2 L_{2}^{4} L_{3}^{4}+2 L_{3}^{4} b_{2} k+2 L_{3}^{4} k\right)+ \\
+3 B_{4,1}^{\star}\left(k \Omega_{20}^{2}\left(L_{2}^{4}\right)^{2}+2 b_{4} k \Omega_{20}^{2}\left(L_{2}^{4}\right)^{2}-2 L_{2}^{4} L_{3}^{4} b_{4}-2 L_{3}^{4} b_{4} k-2 L_{5}^{4} b_{4} k-2 L_{5}^{4} k\right)+ \\
+12 F_{1,1}^{\star} L_{1}^{4} L_{2}^{4}+6 F_{3,1}^{\star}\left(3 L_{2}^{4} L_{3}^{4} b_{3}+L_{2}^{4} L_{3}^{4}-3 L_{3}^{4} b_{3} k-2 L_{3}^{4} k\right)-6 F_{5,1}^{\star}\left(3 L_{5}^{4} b_{5} k+L_{5}^{4} k\right)+ \\
\left.+6 G_{3,1}^{\star}\left(L_{2}^{4} L_{3}^{4}-L_{3}^{4} k\right)-6 G_{5,1}^{\star} L_{5}^{4} k\right)
\end{gathered}
$$

and all the constants with $\star$ are obtained from their counterparts through division by $C_{4,0}$.

\section{NUMERICAL RESULTS}

Consider a five-layered laminate studied in the previous section with the layers of same thickness $h$, i.e. $h_{i}=h, i=1,2, \ldots, 5$. Then, we have from (17) and (24) for eigenfrequencies, and from (19), (20), and (21) for eigenform at $k=1$ and $k=-1$, respectively

$$
\Omega_{2}^{2}=\varepsilon\left(1-\frac{\varepsilon}{6}\left(5 c^{2}+2\right)+\ldots\right),
$$


with

$$
\begin{aligned}
& u_{1}=Z_{1}+\frac{\varepsilon}{6} Z_{1}\left(-Z_{1}^{2} c^{2}+c^{2}+24\right)+\ldots \\
& u_{2}=1+\frac{\varepsilon}{2}\left(-Z_{2}^{2}+4 Z_{2}+5\right)+\ldots \\
& u_{3}=1+\frac{\varepsilon}{2}\left(-c^{2} Z_{3}^{2}+5 c^{2} Z_{3}-6 c^{2}+9\right)+\ldots, \\
& u_{4}=1-\frac{\varepsilon}{2} Z_{4}\left(Z_{4}-6\right)+\ldots, \\
& u_{5}=-Z_{5}+5+\frac{\varepsilon}{6}\left(c^{2} Z_{5}^{3}-15 c^{2} Z_{5}^{2}-24 Z_{5}+74 Z_{5} c^{2}-120 c^{2}+120\right)+\ldots
\end{aligned}
$$

and

$$
\Omega_{2}^{2}=\varepsilon\left(3-\frac{\varepsilon}{2}\left(3 c^{2}+2\right)+\ldots\right)
$$

with

$$
\begin{aligned}
& u_{1}=-Z_{1}+\frac{\varepsilon}{2} Z_{1}\left(Z_{1}^{2} c^{2}-c^{2}-40\right)+\ldots, \\
& u_{2}=-1+\frac{\varepsilon}{2}\left(3 Z_{2}^{2}-8 Z_{2}-35\right)+\ldots, \\
& u_{3}=2 Z_{3}-5+\frac{\varepsilon}{2}\left(-2 c^{2} Z_{3}^{3}+15 c^{2} Z_{3}^{2}-37 c^{2} Z_{3}+78 Z_{3}+30 c^{2}-195\right)+\ldots, \\
& u_{4}=1-\frac{\varepsilon}{2} Z_{4}\left(3 Z_{4}-22\right)+\ldots, \\
& u_{5}=-Z_{5}+5+\frac{\varepsilon}{2}\left(c^{2} Z_{5}^{3}-15 c^{2} Z_{5}^{2}+74 Z_{5} c^{2}-40 Z_{5}-120 c^{2}+200\right)+\ldots
\end{aligned}
$$

The eigenfrequencies, calculated from one-term and two-term asymptotic formulae, see (25) and (27), versus their exact values, for which $\operatorname{det} M=0$ in (30), are presented in the Table. Here and below $\varepsilon=0.01$ and $c=1$.

Comparison of exact and approximate eigenfrequencies

\begin{tabular}{|c|c|c|c|}
\hline$k$ & $\Omega_{2}^{2}=\varepsilon \Omega_{20}^{2}$ & $\Omega_{2}^{2}=\varepsilon\left(\Omega_{20}^{2}+\varepsilon \Omega_{21}^{2}\right)$ & exact value $\Omega_{2}^{2}$ \\
\hline 1 & 0.01 & 0.009883 & 0.009884 \\
\hline-1 & 0.03 & 0.02975 & 0.02975 \\
\hline
\end{tabular}

Fig. 3 demonstrates a good agreement between two-term expansions (26) and (28) and exact solution (29) for both $k= \pm 1$. In this figure the exact eigenforms are normalized by constant $U_{24}\left(U_{24} \approx C_{40}\right)$, see Appendix.
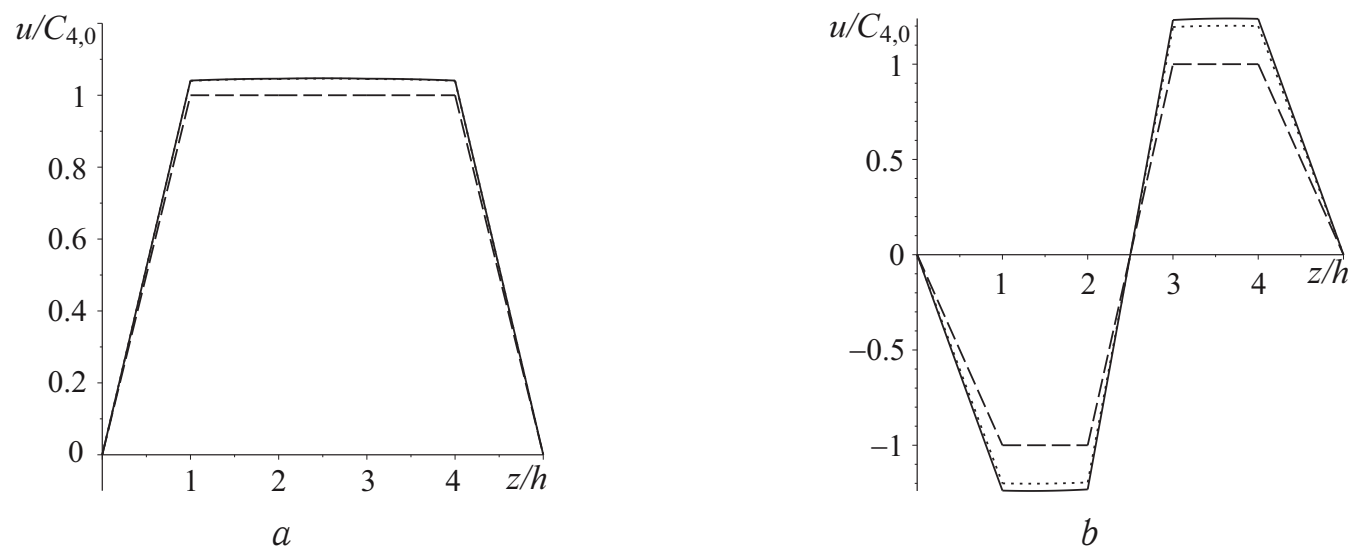

Fig. 3. Asymptotic expansions (26), $k=1(a)$ and (28), $k=-1(b)$ of the eigenforms of a regular five-layered laminate at $\varepsilon=0$ (dashed line) and $\varepsilon=0.01, c=1$ (dotted line) together with exact solution (29) (solid line) 


\section{CONCLUDING REMARKS}

The developed methodology seems to be the initial stage in analysing low-frequency vibrations of strongly inhomogeneous multi-layered structures. The next step, following the evaluation of low-frequency thickness resonances, is concerned with the derivation of multi-mode polynomial approximations of the original dispersion relations, similarly to [6]. The final expected outcome should involve two-dimensional equations of motion supplied with appropriate boundary conditions at the edges. The proposed approach may be easily extended to layered anisotropic and pre-stressed structures, previously investigated only for homogeneous and non-contrast configurations, e.g. see [16-18]. Certainly, various generalisations dealing with another scaling for problem parameters are also possible.

Acknowledgements: This work was supported by the grant J2-9224 from the Slovenian Research Agency and also by Program OP20.00362 EVA4GREEN. L. A. Prikazchikova acknowledges support by the Faculty Award by Keele University, UK.

\section{APPENDIX}

The solution of equations (5) for a five-component laminate can be written as

$$
u_{i}=U_{i 1} \sin \left(\Omega_{i} Z_{i}\right)+U_{i 2} \cos \left(\Omega_{i} Z_{i}\right), \quad i=1, \ldots, 5,
$$

where $U_{i 1}$ and $U_{i 2}$ are constants. On substituting (29) into continuity conditions (9), (10) and boundary conditions (8), we arrive at the eigenvalue problem

$$
\mathrm{M} \cdot \mathrm{U}=0
$$

where $\mathrm{U}=\left(U_{11}, U_{12}, U_{21}, U_{22}, U_{31}, U_{32}, U_{41}, U_{42}, U_{51}, U_{52}\right)^{\mathrm{T}}$, is an eigenvector and $\mathrm{M}$ is a $10 \times 10$ matrix with non-zero components given by

$$
\begin{gathered}
M_{12}=1, \quad M_{21}=\sin \left(\Omega_{1}\right), \quad M_{22}=\cos \left(\Omega_{1}\right), \quad M_{23}=-\sin \left(\Omega_{2} b_{2}\right), \quad M_{24}=-\cos \left(\Omega_{2} b_{2}\right), \\
M_{33}=\sin \left(\Omega_{2}\left(b_{2}+1\right)\right), \quad M_{34}=\cos \left(\Omega_{2}\left(b_{2}+1\right)\right), \quad M_{35}=-\sin \left(\Omega_{3} b_{3}\right), \quad M_{36}=-\cos \left(\Omega_{3} b_{3}\right), \\
M_{45}=\sin \left(\Omega_{3}\left(b_{3}+1\right)\right), \quad M_{46}=\cos \left(\Omega_{3}\left(b_{3}+1\right)\right), \quad M_{47}=-\sin \left(\Omega_{4} b_{4}\right), \quad M_{48}=-\cos \left(\Omega_{4} b_{4}\right), \\
M_{57}=\sin \left(\Omega_{4}\left(b_{4}+1\right)\right), \quad M_{58}=\cos \left(\Omega_{4}\left(b_{4}+1\right)\right), \quad M_{59}=-\sin \left(\Omega_{5} b_{5}\right), \quad M_{510}=-\cos \left(\Omega_{5} b_{5}\right), \\
M_{69}=\sin \left(\Omega_{5}\left(b_{5}+1\right)\right), \quad M_{610}=\cos \left(\Omega_{5}\left(b_{5}+1\right)\right), \\
M_{71}=\Omega_{1} \cos \left(\Omega_{1}\right) \varepsilon, \quad M_{72}=-\Omega_{1} \sin \left(\Omega_{1}\right) \varepsilon, \quad M_{73}=-L_{2}^{1} \cos \left(\Omega_{2} b_{2}\right) \Omega_{2}, \quad M_{74}=L_{2}^{1} \sin \left(\Omega_{2} b_{2}\right) \Omega_{2}, \\
M_{83}=\Omega_{2} \cos \left(\Omega_{2}\left(b_{2}+1\right)\right), \quad M_{84}=-\Omega_{2} \sin \left(\Omega_{2}\left(b_{2}+1\right)\right), \quad M_{85}=-L_{3}^{2} \cos \left(\Omega_{3} b_{3}\right) \Omega_{3} \varepsilon, \\
M_{86}=L_{3}^{2} \sin \left(\Omega_{3} b_{3}\right) \Omega_{3} \varepsilon, \quad M_{95}=\Omega_{3} \cos \left(\Omega_{3}\left(b_{3}+1\right)\right) \varepsilon, \quad M_{96}-\Omega_{3} \sin \left(\Omega_{3}\left(b_{3}+1\right)\right) \varepsilon, \\
M_{97}=-L_{4}^{3} \cos \left(\Omega_{4} b_{4}\right) \Omega_{4}, \quad M_{98}=L_{4}^{3} \sin \left(\Omega_{4} b_{4}\right) \Omega_{4}, \quad M_{107}=\Omega_{4} \cos \left(\Omega_{4}\left(b_{4}+1\right)\right), \\
M_{108}=-\Omega_{4} \sin \left(\Omega_{4}\left(b_{4}+1\right)\right), \quad M_{109}=-L_{5}^{4} \cos \left(\Omega_{5} b_{5}\right) \Omega_{5} \varepsilon, \quad M_{1010} L_{5}^{4} \sin \left(\Omega_{5} b_{5}\right) \Omega_{5} \varepsilon .
\end{gathered}
$$

\section{References}

1. Kossovich L. Yu. Nestacionarnye zadachi teorii uprugih tonkih obolochek [Nonstationary problems in the theory of elastic thin shells]. Saratov, Saratov Univ. Press, 1986 (in Russian).

2. Kaplunov J. D., Kossovich L. Y., Nolde E. V. Dynamics of thin walled elastic bodies. Academic Press, 1998. 
3. Beresin V. L., Kossovich L. Y., Kaplunov J. D. Synthesis of the dispersion curves for a cylindrical shell on the basis of approximate theories. Journal of sound and vibration, 1995, vol. 186, no. 1, pp. 37-53. DOI: https://doi.org/10.1006/jsvi.1995.0432

4. Le K. C. Vibrations of shells and rods. Berlin, Springer, 1999.

5. Berdichevsky V. Л. Variacionnye principy mekhaniki sploshnoj sredy [Variational Principles of Continuum Mechanics]. Moscow, Nauka, Glav. red. fiz.-mat. lit., 1983 (in Russian).

6. Kaplunov J., Prikazchikov D. A., Prikazchikova L. A. Dispersion of elastic waves in a strongly inhomogeneous three-layered plate. International Journal of Solids and Structures, 2017, vol. 113-114, pp. 169-179. DOI: https://doi.org/10.1016/j.ijsolstr.2017.01.042

7. Kossovich L. Yu., Shevtsova Yu. V. Asymptotic approximations of three-dimensional dynamic equations of elasticity theory in case of two-layered plates. Problems of strenght and plasticity, 2005, vol. 76, pp. 102-111 (in Russian). DOI: https://doi.org/10.32326/18149146-2005-67-1-102-110

8. Prikazchikova L., Ece Aydın Y., Erbaş B., Kaplunov J. Asymptotic analysis of an anti-plane dynamic problem for a three-layered strongly inhomogeneous laminate. Mathematics and Mechanics of Solids, 2018. DOI: https://doi.org/10.1177/1081286518790804

9. Kaplunov J., Prikazchikov D., Sergushova O. Multi-parametric analysis of the lowest natural frequencies of strongly inhomogeneous elastic rods. Journal of Sound and Vibration, 2016, vol. 366, pp. 264-276. DOI: https://doi.org/10.1016/j.jsv.2015.12.008

10. Vinson J. R. The behavior of sandwich structures of isotropic and composite materials. CRC Press, 1999.

11. Ivanov I. V. Analysis, modelling, and optimization of laminated glasses as plane beam. International Journal of Solids and Structures, 2006, vol. 43, no. 22-23, pp. 6887-6907. DOI: https://doi.org/10.1016/j.ijsolstr.2006.02.014

12. Schulze S.-H., Pander M., Naumenko K., Altenbach H. Analysis of laminated glass beams for photovoltaic applications. International Journal of Solids and Structures, 2012, vol. 49, pp. 2027-2036. DOI: https://doi.org/10.1016/j.ijsolstr.2012.03.028

13. Lee P., Chang N. Harmonic waves in elastic sandwich plates. Journal of Elasticity, 1979, vol. 9, pp. 51-69. DOI: https://doi.org/10.1007/BF00040980

14. Kaplunov J. D. Long-wave vibrations of a thinwalled body with fixed faces. The Quarterly Journal of Mechanics and Applied Mathematics, 1995, vol. 48, no. 3, pp. 311-327. DOI: https://doi.org/10.1093/qjmam/48.3.311

15. Kaplunov J. D., Nolde E. V. Long-wave vibrations of a nearly incompressible isotropic plate with fixed faces. The Quarterly Journal of Mechanics and Applied Mathematics, 2002, vol. 55, no. 3, pp. 345-356. DOI: https://doi.org/10.1093/qjmam/55.3.345

16. Kaplunov J. D., Kossovich L. Yu., Rogerson G. A. Direct asymptotic integration of the equations of transversely isotropic elasticity for a plate near cut-off frequencies. Quarterly Journal of Mechanics and Applied Mathematics, 2000, vol. 53, no. 2, pp. 323-341.

17. Nolde E. V., Rogerson G. A. Long wave asymptotic integration of the governing equations for a pre-stressed incompressible elastic layer with fixed faces. Wave Motion, 2002, vol. 36, no. 3, pp. 287-304. DOI: https://doi.org/10.1016/S0165-2125(02)00017-3

18. Rogerson G. A., Sandiford K. J., Prikazchikova L. A. Abnormal long wave dispersion phenomena in a slightly compressible elastic plate with non-classical boundary conditions. International Journal of Non-Linear Mechanics, 2007, vol. 42, no. 2, pp. 298-309. DOI: https://doi.org/10.1016/j.ijnonlinmec.2007.01.005

\section{Cite this article as:}

Kaplunov J. D., Prikazchikova L. A. Low-Frequency Vibration Modes of Strongly Inhomogeneous Elastic Laminates. Izv. Saratov Univ. (N. S.), Ser. Math. Mech. Inform., 2018, vol. 18, iss. 4, pp. 447-457. DOI: https://doi.org/10.18500/1816-9791-2018-18-4-447-457 


\title{
ДЛИННОВОЛНОВЫЕ МОДЫ КОЛЕБАНИЙ СИЛЬНО НЕОДНОРОДНЫХ УПРУГИХ СЛОИСТЫХ КОНСТРУКЦИЙ
}

\begin{abstract}
Ю. Д. Каплунов, Л. А. Приказчикова
Каплунов Юлий Давидович, доктор фризико-математических наук, просрессор, Школа компьютерных наук и математики, Университет г. Киль, Киль, Стафрфордшир, ST5 5BG, Великобритания, j.kaplunov@keele.ac.uk

Приказчикова Людмила Анатольевна, кандидат фризико-математических наук, Школа компьютерных наук и математики, Университет г. Киль, Киль, Стафррордшир, ST5 5BG, Великобритания, I.prikazchikova@keele.ac.uk

В статье изучается динамика тонких многослойных конструкций с контрастными «мягкими» и «жесткими» слоями. Разработана асимптотическая процедура для анализа малых частот среза. Получено полиномиальное частотное уравнение, а также линейные уравнения для собственных фрорм толщинных колебаний. В случае пятислойной пластины с зажатыми лицевыми поверхностями выведены двухчленные асимптотические разложения для собственных частот и фрорм, которые сопоставляются с точным решением исходной задачи о свободных толщинных колебаниях.
\end{abstract}

Ключевые слова: асимптотика, контраст, слоистые упругие конструкции, толщинные колебания, малые частоты, срез.

Благодарности. Статья выполнена при финансовой поддержке Словенского исследовательского агентства (проект № J2-9224) и программы OP20.00362 EVA4GREEN. Работа Л. А. Приказчиковой выполнена при финансовой поддержке премии факультета Университета Киля, Великобритания.

\section{Образец для цитирования:}

Kaplunov J. D., Prikazchikova L. A. Low-Frequency Vibration Modes of Strongly Inhomogeneous Elastic Laminates [Каплунов Ю. Д., Приказиикова Л. А. Длинноволновые моды колебаний сильно неоднородных упругих слоистых конструкций] // Изв. Сарат. ун-та. Нов. сер. Сер. Математика. Механика. Информатика. 2018. Т. 18, вып. 4. C. 447-457. DOI: https://doi.org/10.18500/1816-9791-2018-18-4-447-457 